\title{
In Vitro Effects of Paclitaxel and Cremophor EL on Human Riboflavin Transporter SLC52A2
}

\author{
Hitomi Hino, ${ }^{a, \uparrow}$ Mingdong Yang, ${ }^{a}$ Pooja Dalvi, ${ }^{a}$ Tongtong Chen, ${ }^{a, \downarrow}$ Linda Sun,,${ }^{a}$ \\ Patricia A. Harper, ${ }^{b}$ and Shinya Ito ${ }^{*, a, b, c}$ \\ ${ }^{a}$ Translational Medicine Program, Research Institute, The Hospital for Sick Children; 686 Bay St., Toronto, Ontario \\ M5G 0A4, Canada: ${ }^{b}$ Department of Pharmacology and Toxicology, University of Toronto; 1 King's College Cir, \\ Toronto, Ontario M5S 1A8, Canada: and ${ }^{c}$ Department of Paediatrics, The Hospital for Sick Children, University of \\ Toronto; 555 University Avenue, Toronto, Ontario M5G 1X8, Canada. \\ Received May 8, 2019; accepted October 4, 2019
}

Paclitaxel, a mitotic inhibitor with anti-cancer effects, is dissolved in Cremophor EL (CrEL). However, peripheral neuropathy is a known side effect. As one of the mechanisms of the neuropathy, mitochondrial dysfunction has been proposed, while peroxidation products are involved in the cause of CrEL-induced neurotoxicity. Riboflavin is an essential nutrient required for ATP production in mitochondria and has an antioxidant role as a coenzyme for glutathione. Therefore, riboflavin transporters might play a key role to mitigate neuropathy. However, it is unclear whether paclitaxel and CrEL affect these transporters. In this study, human riboflavin transporter SLC52A2 was used to analyze the effects of paclitaxel and CrEL. CrEL, but not paclitaxel, inhibited uptake of riboflavin in human embryonic kidney 293 cells transfected with the SLC52A2 expression vector, suggesting that altered riboflavin disposition may be involved in the pathogenesis of paclitaxel/CrEL toxicity.

Key words Cremophor EL; riboflavin; SLC52A2; paclitaxel; peripheral neuropathy

\section{INTRODUCTION}

Paclitaxel has a broad range of anti-cancer activities against solid tumors such as breast, stomach, lung, head and neck, prostate and gynecological malignancies. ${ }^{1)}$ Following its binding to $\beta$-tubulin, paclitaxel destabilizes microtubule dynamics, inhibits $\mathrm{G} 2 / \mathrm{M}$ phase transition in the cell cycle, and then activates the mitochondrial apoptotic pathway. ${ }^{2}$

Mitochondrial dysfunction is a major mechanism for not only anti-cancer effects, but also peripheral neuropathy as a side effect. Previous studies suggest that an abnormality in axonal mitochondria of sensory nerves induced by paclitaxel in Cremophor EL (CrEL) as the vehicle contributes to pain in rats. ${ }^{3,4}$ Swelling and vacuolation of mitochondria and decreased ATP production in the sensory nerve caused by paclitaxel in CrEL were shown in these studies. Mitotoxic effects on sensory neurons give rise to a persistent energy deficit. ${ }^{5)}$ In particular, neuronal cells require energy from ATP to carry neurotransmitters and mitochondria through axons. $\left.{ }^{6}\right)$ The axons of neurons are especially susceptible to damage related to mitochondrial dysfunction.

Riboflavin is essential for many enzymatic reactions that occur in mitochondria and contributes to ATP production.

\footnotetext{
${ }^{\dagger}$ Present address: Department of Pediatrics and Regional Medical Play Laboratory, Ehime University Graduate School of Medicine; Shitsukawa, Toon, Ehime 791-0295, Japan.

ॠ Present address: Department of Pharmacology and Toxicology, University of Toronto; Medical Sciences Building, 1 King's College Circle, Room 4207, Toronto, Ontario M5S 1A8, Canada.

${ }^{\S}$ Present address: Molecular, Genetic and Population Health Sciences of Edinburgh Medical School; Old College, South Bridge, Edinburgh EH8 9YL, Scotland, U.K.
}

Riboflavin uptake into cells is mediated by SLC52A transporters. Recently, three types of the transporter have been discovered. $^{7-9)}$ SLC52A2, which is one of the riboflavin transporters in the brain, might play important roles in the neuronal system. ${ }^{10)}$

Hereditary riboflavin transporter deficiency induces axonal neuropathy that has the same pathology as peripheral neuropathy induced by paclitaxel in CrEL. Paclitaxel is hydrophobic and must be dissolved in solvents such as CrEL, a vehicle that causes axonal peripheral neuropathy as a side effect. ${ }^{11)}$ Residual unsaturated fatty acids, which are peroxidation products, are one of the causes of neurotoxicity induced by CrEL. ${ }^{12)}$ Riboflavin also has an antioxidant role that contributes to the glutathione cycle.

Although treatments aimed at protecting or restoring mitochondrial functions and anti-oxidation may have general utility, and riboflavin is a source for energy production in mitochondria and an antioxidant, the effect of riboflavin on peripheral neuropathy has not been studied. The present study assessed the effect of paclitaxel and CrEL on riboflavin transporters transfected into human embryonic kidney (HEK) 293 cells.

\section{MATERIALS AND METHODS}

Cell Line and Reagents HEK293 cells (American Tissue Culture Collection, Manassas, VA, U.S.A.) were seeded at $2.5 \times 10^{5}$ cells per well in 12 -well poly-D-lysine-coated plates (Corning, Corning, NY, U.S.A.) and grown in Dulbecco's modified Eagle's medium supplemented with $10 \%$ fetal bovine serum. Cells were maintained in a humidified incubator at $37^{\circ} \mathrm{C}$ with $5 \% \mathrm{CO}_{2}$.

Riboflavin Uptake Assay pCMV6 (cat. no. pCMV6KN)

* To whom correspondence should be addressed. e-mail: shinya.ito@sickkids.ca 

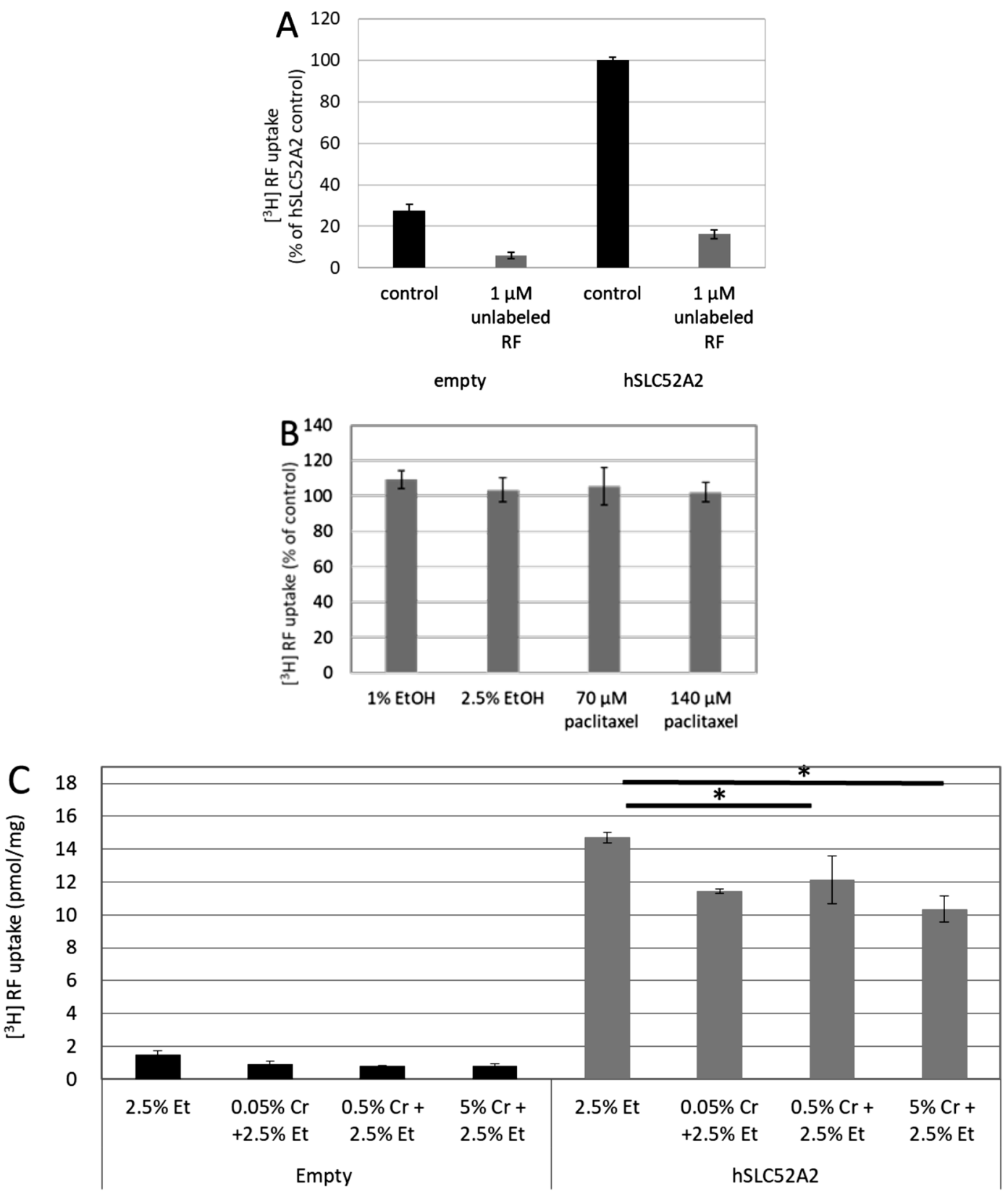

Fig. 1. CrEL Inhibited Uptake of ${ }^{3} \mathrm{H}-$ Riboflavin in HEK293 Transfected with Human SLC52A2

A: Riboflavin uptake was much higher in cells transfected riboflavin transporter (empty: $1.58 \pm 0.18 \mathrm{pmol} / \mathrm{mg}$ protein; hSLC52A2 transfected: $5.79 \pm 0.08 \mathrm{pmol} / \mathrm{mg}$ protein), and it was suppressed by excess amount of unlabeled riboflavin, suggesting functional overexpression of the transporter. B: Ethanol (1 and $2.5 \%)$ and paclitaxel (70 and $140 \mu \mathrm{M}$ ) did not inhibit the uptake. Note that $1 \% \mathrm{CrEL}+1 \%$ EtOH was added as a vehicle control for paclitaxel. The amount of uptake of riboflavin for each conditions were $12.74 \pm 0.65,10.60 \pm 0.74,11.67 \pm 1.22$ and $8.84 \pm 0.49 \mathrm{pmol} / \mathrm{mg}$ protein, respectively. $\mathrm{C}: 5$ and $0.5 \%$ CrEL in $2.5 \%$ ethanol inhibited the uptake significantly through the human transporter $(* ; p<0.05)$. Mean \pm S.D.; $n=4-12$.

and pCMV6-hSLC52A2 (cat. no. SC108833) were purchased from Origene Technologies (Rockville, MD, U.S.A.).

HEK293 cells were transfected using a 3:1 ratio of Fugene HD (Roche) to DNA with $1 \mu \mathrm{g}$ plasmid per well as described previously. ${ }^{10)}$

Forty-eight hours after transfection, HEK293 cells were preincubated with $\mathrm{N} 1$ buffer $(25 \mathrm{mM} N$-(2-hydroxyethyl)piperazine- $N$ '-2-ethanesulfonic acid (HEPES), $125 \mathrm{mM} \mathrm{NaCl}$, $4.8 \mathrm{mM} \mathrm{KCl}, 1.2 \mathrm{mM} \mathrm{CaCl}_{2}, 1.2 \mathrm{mM} \mathrm{MgSO}_{4}, 1.2 \mathrm{mM} \mathrm{K}_{2} \mathrm{HPO}_{4}$, and $5.6 \mathrm{mM}$ D-glucose, $\mathrm{pH} \mathrm{7.5)} \mathrm{for} 1 \mathrm{~h}$ at $37^{\circ} \mathrm{C}$ and then incubated with $\mathrm{N} 1$ buffer containing $5 \mathrm{nM}$ (for Fig. 1A) or $100 \mathrm{nM}$ (for other experiments) ${ }^{3} \mathrm{H}$-labeled riboflavin $\left(\left[{ }^{3} \mathrm{H}\right]\right.$ riboflavin: $6.2 \mathrm{mCi} / \mathrm{mmol}$; Moravek Biochemicals, Brea, CA, U.S.A.) with or without paclitaxel, CrEL, and $\mathrm{EtOH}$ for $10 \mathrm{~min}$ at room temperature.

A paclitaxel solution $(7 \mathrm{mM})$ was prepared from $30 \mathrm{mg}$ paclitaxel dissolved in $5 \mathrm{~mL}$ of $50 \% \mathrm{CrEL}$ and $50 \% \mathrm{EtOH}(1: 1$ $(\mathrm{v} / \mathrm{v}))$. Paclitaxel $(70$ and $140 \mu \mathrm{M})$ was prepared as 1 and $2 \%$ paclitaxel $(7 \mathrm{mM})$ in $50 \% \mathrm{CrEL}$ and $50 \% \mathrm{EtOH}(\mathrm{v} / \mathrm{v})$. Several concentrations of CrEL were prepared in ethanol to examine the effect of CrEL $(0.05,0.5$, and $5 \%(\mathrm{v} / \mathrm{v}))$. The uptake process was terminated by placing the culture plates on ice and washing the cells with ice-cold phosphate buffered saline (PBS) twice. Cells were lysed with $800 \mu \mathrm{L}$ of $0.01 \mathrm{~N} \mathrm{NaOH}$ for $1 \mathrm{~h}$ at $37^{\circ} \mathrm{C}$. The radioactivity in $600 \mu \mathrm{L}$ of cell lysate was determined using a liquid scintillation counter (LS6500; Beckman Coulter, Ramsey, MN, U.S.A.). Protein content in the cell lysate was measured using the bicinchoninic acid assay 
(Thermo Scientific, Ottawa, Canada).

Statistical Analysis Results are expressed as the mean \pm standard deviation (S.D.). Statistical significance was tested by Kruskal-Wallis test after determining the normality (Shapiro-Wilk test) of experimental data. Post-hoc testing was performed by Steel's multiple comparison test. A $p$-value of less than 0.05 was considered as significant. All statistical analyses were performed using EZR software version 1.35 (Saitama Medical Center, Jichi Medical University, Saitama, Japan).

\section{RESULTS}

CrEL, a Vehicle for Paclitaxel, Inhibits Uptake of ${ }^{3} \mathrm{H}$ Riboflavin by HEK293 Cells Transfected with Human SLC52A2 Expression Vectors In HEK293 cells transfected with the human SLC52A2 expression vector (hSLC52A2HEK293), ${ }^{3}$ H-riboflavin uptake was nearly 5-fold higher than those transfected with empty vector (empty-HEK293), and an excess amount of unlabeled riboflavin inhibited the uptake (Fig. 1A), suggesting functional overexpression of SLC52A2 in hSLC52A2-HEK293 cells. We then examined whether ethanol as a component of the paclitaxel/CrEL solution affected uptake of riboflavin into hSLC52A2-HEK293. Ethanol at 1 and $2.5 \%$, and paclitaxel at 70 and $140 \mu \mathrm{M}$ in CrEL did not affect uptake (Fig. 1B). In contrast, CrEL at 0.5 and $5 \%$ in $2.5 \%$ ethanol inhibited riboflavin uptake significantly in hSLC52A2HEK293 ( $n=6$, Fig. 1C, *; $p<0.05$ ). Although riboflavin uptake was observed in empty-HEK293, CrEL inhibition of the uptake was clearly small compared with hSLC52A2-HEK293 (Fig. 1C).

\section{DISCUSSION}

CrEL, a widely used formulation vehicle, is a biologically and pharmacologically active ingredient of various clinically available drugs. A single administration dose of $\mathrm{CrEL}$ as the vehicle for paclitaxel is one of the largest amounts among several drugs. ${ }^{13)}$ Myelosuppression and neuropathy are major side effects of paclitaxel formulated with CrEL. Recently, supportive therapy has been established for myelosuppression, but neurotoxicity remains the main reason to decrease the total cumulative dose administered, which may affect the therapeutic outcome. ${ }^{14)}$ It has also been reported that neurotoxicity is induced by CrEL alone. ${ }^{13)}$

This study showed that CrEL dose-dependently inhibited uptake of riboflavin by HEK293 cells transfected with the SLC52A2 riboflavin transporter (Fig. 1C). We had previously shown cell membrane localization of SLC52A2 in HEK293 cells, ${ }^{10)}$ which supports validity of this system for SLC52A2mediated membrane transport. After intravenous administration, paclitaxel results in peak serum concentrations of 2.5-10.7 $\mathrm{nM}$, and the associated CrEL peak serum concentration is reportedly in the range of $0.2-0.8 \%(\mathrm{v} / \mathrm{v}) .^{15,16)}$ Windebank et al. showed $0.01-1 \%$ concentration of CrEL inhibited neurite outgrowth of dorsal root ganglion cells in vitro. ${ }^{17)}$ Therefore $0.05-5 \%$ of CrEL was used in our experiments. In our study, we observed a decline of riboflavin uptake at 0.5 and $5 \% \mathrm{CrEL}$. As compared to total activity of riboflavin uptake, the inhibitory effect by clinical dose of CrEL is weak. However, there is little information about the pharmacokinet- ics of CrEL in humans. Because CrEL is a nonionic detergent, it is likely to be distributed widely in body tissues, and it may accumulate in lipid-rich areas such as the central nervous system $(\mathrm{CNS})^{17)}$ and the effect of CrEL should not be ignored.

Although a causal link of this phenomenon to paclitaxel/ CrEL-associated neuropathy has yet to be proven, riboflavin may be a potential prophylactic treatment for peripheral neuropathy induced by paclitaxel in CrEL.

The inhibition of riboflavin uptake by CrEL may increase mitochondrial dysfunction induced by paclitaxel. Alteration in energy metabolism due to mitochondria dysfunction is one of the major causes of paclitaxel-induced peripheral neuropathy. ${ }^{18)}$ Neurons require large amounts of energy to transport neuronal transmitters in axons. Flavin mononucleotide (FMN) and FAD, which are derived from riboflavin, are used for the electron transport chain to produce ATP, the primary energy carrier in cells. Riboflavin deficiency may suppress the activity of mitochondria and exacerbate neurotoxicity.

Furthermore, the inhibition of riboflavin uptake by $\mathrm{CrEL}$ may decrease the antioxidant effect through the glutathione redox cycle or other possible mechanisms such as conversion of reduced riboflavin to the oxidized form. ${ }^{19-21)}$ The antioxidant effect by riboflavin might play a role in preventing neurotoxicity.

It still remains unclear whether CrEL specifically inhibits SLC52A2, suppresses global transporter functions, or disrupts the cell membrane. ${ }^{13)}$ SLC52A2 mutation is the cause of Brown-Vialetto-Van Laere (BVVL) syndrome. Supplementation of riboflavin in patients improves the symptoms of axonal peripheral neuropathy. ${ }^{22)}$ Investigation of the mechanism involved in paclitaxel-induced neuropathy may provide evidence for the management of BVVL syndrome with riboflavin.

In conclusion, CrEL is a candidate as an inhibitor of the riboflavin transporter SLC52A2. Supplementation of riboflavin has the potential to prevent neurotoxicity induced by paclitaxel dissolved in CrEL, because riboflavin is a coenzyme in mitochondria and antioxidant. Further investigation is needed to show that inhibition of riboflavin uptake contributes to the mechanism of peripheral neuropathy caused by paclitaxel in $\mathrm{CrEL}$, and that riboflavin improves mitochondrial dysfunction and oxidative stress leading to the neuropathy.

Acknowledgment We thank Mitchell Arico for editing a draft of this manuscript.

Conflict of Interest The authors declare no conflict of interest.

\section{REFERENCES}

1) Khanna C, Rosenberg M, Vail DM. A review of paclitaxel and novel formulations including those suitable for use in dogs. J. Vet. Intern. Med., 29, 1006-1012 (2015).

2) Ferlini C, Cicchillitti L, Raspaglio G, Bartollino S, Cimitan S, Bertucci C, Mozzetti S, Gallo D, Persico M, Fattorusso C, Campiani G, Scambia G. Paclitaxel directly binds to Bcl-2 and functionally mimics activity of Nur77. Cancer Res., 69, 6906-6914 (2009).

3) Flatters SJ, Bennett GJ. Studies of peripheral sensory nerves in paclitaxel-induced painful peripheral neuropathy: evidence for mitochondrial dysfunction. Pain, 122, 245-257 (2006).

4) Zheng H, Xiao WH, Bennett GJ. Functional deficits in peripheral nerve mitochondria in rats with paclitaxel- and oxaliplatin-evoked 
painful peripheral neuropathy. Exp. Neurol., 232, 154-161 (2011).

5) Bennette NB, Eng JF, Dismukes GC. An LC-MS-based chemical and analytical method for targeted metabolite quantification in the model cyanobacterium Synechococcus sp. PCC 7002. Anal. Chem., 83, 3808-3816 (2011)

6) Attwell D, Laughlin SB. An energy budget for signaling in the grey matter of the brain. J. Cereb. Blood Flow Metab., 21, 1133-1145 (2001).

7) Yamamoto S, Inoue K, Ohta KY, Fukatsu R, Maeda JY, Yoshida Y, Yuasa H. Identification and functional characterization of rat riboflavin transporter 2. J. Biochem., 145, 437-443 (2009).

8) Yao Y, Yonezawa A, Yoshimatsu H, Masuda S, Katsura T, Inui $\mathrm{K}$. Identification and comparative functional characterization of a new human riboflavin transporter hRFT3 expressed in the brain. $J$. Nutr., 140, 1220-1226 (2010).

9) Yonezawa A, Masuda S, Katsura T, Inui K. Identification and functional characterization of a novel human and rat riboflavin transporter, RFT1. Am. J. Physiol. Cell Physiol., 295, C632-C641 (2008).

10) Wu AM, Dedina L, Dalvi P, Yang M, Leon-Cheon J, Earl B, Harper PA, Ito S. Riboflavin uptake transporter Slc52a2 (RFVT2) is upregulated in the mouse mammary gland during lactation. Am. J. Physiol. Regul. Integr. Comp. Physiol., 310, R578-R585 (2016).

11) $\mathrm{Du} \mathrm{AW,} \mathrm{Lu} \mathrm{H,} \mathrm{Stenzel} \mathrm{MH.} \mathrm{Core-cross-linking} \mathrm{accelerates} \mathrm{anti-}$ tumor activities of paclitaxel-conjugate micelles to prostate multicellular tumor spheroids: a comparison of $2 \mathrm{D}$ and 3D models. Biomacromolecules, 16, 1470-1479 (2015).

12) Anthony EW, Nevins ME. Anxiolytic-like effects of $N$-methyl-Daspartate-associated glycine receptor ligands in the rat potentiated startle test. Eur. J. Pharmacol., 250, 317-324 (1993).

13) Gelderblom H, Verweij J, Nooter K, Sparreboom A, Verweij J, Nooter K, Sparreboom A. Cremophor EL: the drawbacks and ad- vantages of vehicle selection for drug formulation. Eur. J. Cancer, 37, 1590-1598 (2001)

14) Velasco R, Bruna J. Taxane-induced peripheral neurotoxicity. Toxics, 3, 152-169 (2015).

15) Meerum Terwogt J, van Tellingen O, Nannan Panday VR, Huizing MT, Schellens JH, ten Bokkel Huinink WW, Boschma MU, Giaccone G, Veenhof CH, Beijnen JH. Cremophor EL pharmacokinetics in a phase I study of paclitaxel (Taxol) and carboplatin in non-small cell lung cancer patients. Anticancer Drugs, 11, 687-694 (2000).

16) $\overline{\mathrm{Wu}} \mathrm{D}$, Liang Y, Liu Q. Therapeutic drug monitoring of paclitaxel (Zisu) in cancer patients. J. Environ. Pathol. Toxicol. Oncol., 19, 77-80 (2000)

17) Windebank AJ, Blexrud MD, de Groen PC. Potential neurotoxicity of the solvent vehicle for cyclosporine. J. Pharmacol. Exp. Ther., 268, 1051-1056 (1994).

18) Carozzi VA, Canta A, Chiorazzi A. Chemotherapy-induced peripheral neuropathy: What do we know about mechanisms? Neurosci. Lett., 596, 90-107 (2015).

19) Rao PV, Bhat KS. Influence dietary riboflavin deficiency on lenticular glutathione redox cycle, lipid peroxidation, and free radical scavengers in the rat. J. Clin. Biochem. Nutr., 6, 195-204 (1989).

20) Taniguchi M, Hara T. Effects of riboflavin and selenium deficiencies on glutathione and its relating enzyme activities with respect to lipid peroxide content of rat livers. J. Nutr. Sci. Vitaminol., 29, 283-292 (1983)

21) Ashoori M, Saedisomeolia A. Riboflavin (vitamin $\mathrm{B}_{2}$ ) and oxidative stress: a review. Br. J. Nutr., 111, 1985-1991 (2014).

22) Foley AR, Menezes MP, Pandraud A, et al. Treatable childhood neuronopathy caused by mutations in riboflavin transporter RFVT2. Brain, 137, 44-56 (2014). 